\title{
Quantum Geometry and Quantum Mechanics of Integrable Systems
}

\author{
M. V. Karasev \\ Moscow Institute of Electronics and Mathematics
}

\begin{abstract}
Quantum integrable systems and their classical counterparts are considered. We show that the symplectic structure and invariant tori of the classical system can be deformed by a quantization parameter $\hbar$ to produce a new (classical) integrable system. The new tori selected by the $\hbar$-equidistance rule represent the spectrum of the quantum system up to $O\left(\hbar^{\infty}\right)$ and are invariant under quantum dynamics in the long-time range $O\left(\hbar^{-\infty}\right)$. The quantum diffusion over the deformed tori is described. The analytic apparatus uses quantum action-angle coordinates explicitly constructed by an $\hbar$-deformation of the classical actionangles.
\end{abstract}

\section{Introduction}

The most elementary and fundamental systems in quantum mechanics are integrable systems.

A quantum integrable system is a complete set of commuting operators whose classical counterpart (arising as the quantization parameter $\hbar$ tends to zero) is a classical integrable system, i.e., admits a complete set of functions in involution on a symplectic manifold. The joint level surfaces of these functions are assumed to be compact, and thus they are organized into the classical Liouville-Arnold tori fibration [1, 2].

One of basic problems is as follows: How are the spectrum and the time evolution of a quantum integrable system related to the geometry and dynamics of the corresponding classical integrable system?

The well-known semiclassical theory [3] enables one to compute, approximately as $\hbar \rightarrow 0$, the characteristics of quantum systems with many degrees of freedom using the objects of classical mechanics (symplectic geometry). This geometric description works for processes which are not long-time and not long-distance, until the quantum diffusion becomes significant. At long scales $\sim \hbar^{-1}$, the classical geometry is not adequate. In particular, in the integrable case, the classical tori are completely destroyed under longtime evolution by quantum diffusion. The classical symplectic geometry fails as well in attempts to describe higher $\hbar$-corrections to the semiclassical spectrum.

As an important example, one can mention a broad class of systems standing just in-between "quantum" and "classical" ones, like nanosystems, where spatial sizes are 
comparable with the effective wave length. For such systems, the quantization parameter $\hbar$ is not very small, say, $\hbar \sim 1 / 3$ (in dimensionless units), and thus the critical "long" time or distance scale $1 / \hbar$ at which the semiclassical theory fails is actually not long at all and is practically needed to study the system. Therefore, it turns out that the classical symplectic geometry fails to be applicable to nanosystems of this kind.

Natural questions arise. Is there a "quantum" symplectic geometry which works for nanosystems? Can a quantum geometry of this kind be achieved using an $\hbar$ deformation of the classical geometry? More precisely:

Is it possible to deform the original symplectic structure and the set of functions in involution in such a way that the corresponding deformed classical integrable system becomes equivalent to a given quantum integrable system? Here "equivalent" means that the deformed tori selected by the $\hbar$-equidistance rule are invariant under the quantum dynamics and represent the spectrum of the quantum system, as well as its evolution.

Actually, if we remove the word "deformed" from this question, then we face a way of dealing with quantum systems as if these were classical systems restricted to some phase-space grids subjected to quantization rules. This coincides with Planck's basic idea [4] of the old quantum mechanics which dominated between 1905 and 1925, before the Heisenberg and Schrödinger discoveries. Thus, in a sense, the above questions return us back to the "naive" concept of old quantum mechanics.

In the present paper, we show that an answer to these questions is affirmative up to $O\left(\hbar^{\infty}\right)$; moreover, all the deformations mentioned above are achieved by geometrically invariant formulas in regular domains (fibered into tori of some dimension). Thus, we claim that the quantum and the classical inegrability conditions are $\hbar^{\infty}$-equivalent in regular domains. This equivalence implies that the regular part of the spectrum, up to $O\left(\hbar^{\infty}\right)$, and the regular evolution at long-time range $\sim \hbar^{-\infty}$ of the quantum integrable system can be described by using "quantum" geometric objects: the deformed symplectic structure, the deformed invariant tori, the deformed frequencies of multiperiodic rotation, and the diffusion tensor along the tori. These objects are computed by a simple explicit algorithm.

The deformed $\hbar$-equidistant tori are the actual quantum dynamical objects replacing Planck's classical torus grids. The deformed symplectic form vanishes on the deformed tori, and thus these submanifolds are of Einstein-Maslov type [5, 3]. However, the Hamiltonians of the original quantum integrable system are not constant on these deformed tori. The actual quantum energy functions representing the energy levels (spectrum) are obtained by a deformation of the Hamiltonians, and the deformation takes into account the quantum diffusion. The energy functions are in involution with respect to the deformed Poisson bracket.

Thus, it becomes possible in a sense to replace the commutator by a bracket to meet Dirac's ideas in quantization theory [6], as well as to reconcile Bohr's correspondence principle with quantum uncertainty up to $O\left(\hbar^{\infty}\right)$.

One can conclude that, in the framework of regular integrable systems and their perturbations, and up to accuracy $O\left(\hbar^{\infty}\right)$, quantum mechanics turns out to be classical mechanics based on quantum geometry. In particular, nanosystems that are integrable or nearly integrable can be treated up to $O\left(\hbar^{\infty}\right)$ as classical systems with respect to a quantum nano-geometry.

Remark. The term "geometry" is applied in this paper in its immediate meaning, 
without algebraic extensions. The quantum geometric objects are distinct from noncommutative objects or geometric operators used in modern quantum theories. The adjective "quantum" is also used in its original sense, as a synonym of " $\hbar$-discretized" and "corresponding to the classical one as $\hbar \rightarrow 0$, , like in quantization theory [7-11] without involving the sense of uncertainty or Hilbert space extension.

\section{Quantum symplectic structure}

In this section, we show explicit formulas for the first quantum corrections to classical symplectic geometry over a flat space. The general algorithm is described in Section 4.

Assume that the algebra of quantum observables is realized by symmetrized (Weyl ordered) functions $\widehat{A}=A(q, \widehat{p})$ of the generators $q=\left(q^{1}, \ldots, q^{n}\right)$ and $\widehat{p}=-i \hbar \partial / \partial q$, where $q^{j}$ stand for the Euclidean coordinates on $\mathbb{R}^{n}$. The classical observables $A=$ $A(q, p)$ are functions on $T^{*} \mathbb{R}^{n}$, which depend on the quantization parameter in general (this dependence is not reflected in the notation).

Let a quantum integrable system be determined by a set of commuting self-adjoint operators $\widehat{H}_{j}(j=1, \ldots, n)$. Thus,

$$
\left[\widehat{H}_{j}, \widehat{H}_{k}\right]=0
$$

In the $\hbar=0$ limit, the functions

$$
H_{j}^{0} \stackrel{\text { def }}{\rightarrow}=\left.H_{j}\right|_{\hbar=0}, \quad\left\{H_{j}^{0}, H_{k}^{0}\right\}=0
$$

determine a classical integrable system on $T^{*} \mathbb{R}^{n}$ with action-angle coordinates

$$
s=\left(s_{1}, \ldots, s_{n}\right) \quad \text { and } \quad \tau=\left(\tau^{1}, \ldots, \tau^{n}\right), \quad 0 \leq \tau^{j} \leq 2 \pi
$$

The classical Hamiltonians $H_{j}^{0}$ are functions on actions only. Consider a domain in $T^{*} \mathbb{R}^{n}$ on which the Hamiltonians $H_{j}^{0}$ are independent, and thus the tori $\{s=$ const $\}$ are of the same (maximal) dimension $n$ (for details, see [12]).

The classical symplectic form is

$$
\omega \stackrel{\text { def }}{\rightarrow}=d p \wedge d q=\frac{1}{2} J d X \wedge d X, \quad X \stackrel{\text { def }}{\rightarrow}=(\tau, s) .
$$

Here $X$ is regarded as a $2 n$-dimensional vector function and

$$
J=\left(\begin{array}{cc}
0 & I \\
-I & 0
\end{array}\right)
$$

stands for the standard symplectic $2 n \times 2 n$ matrix with zero and identity $n \times n$ blocks.

Define the quantum deformation of the symplectic form (2.3) up to $O\left(\hbar^{4}\right)$ by the following formula:

$$
\omega^{\hbar} \stackrel{\text { def }}{\rightarrow}=\omega+\frac{\hbar^{2}}{2} J\langle\langle X \stackrel{\otimes}{\rightarrow}, X\rangle\rangle J d X \wedge d X+O\left(\hbar^{4}\right) .
$$

Here the $2 n \times 2 n$ matrix

$$
\langle\langle X \stackrel{\otimes}{\rightarrow}, X\rangle\rangle
$$


is composed of the components of the vector function $X$, see (2.3), and the bidifferential operation $\langle\langle\cdot, \cdot\rangle\rangle$ is given by

$$
\left\langle\langle A, B\rangle \stackrel{\text { def }}{\rightarrow}=-\frac{1}{24} D^{3} A \cdot J \otimes J \otimes J \cdot D^{3} B,\right.
$$

where $D$ stands for the derivatives with respect to the Euclidean coordinates on $T^{*} \mathbb{R}^{n}$. This is the very operation entering the $\hbar$-expansion of quantum commutators,

$$
\frac{i}{\hbar}[\widehat{A}, \widehat{B}]=\widehat{\{A, B\}}-\hbar^{2}\langle\widehat{\langle A, B\rangle}\rangle+O\left(\hbar^{4}\right)
$$

The classical form (2.3) vanishes on the classical tori $\{s=$ const $\}$. Now we introduce new tori annihilating the quantum form (2.4).

To each Hamiltonian $H_{j}$, one can assign the energy function

$$
H_{j}^{\hbar} \stackrel{\text { def }}{\rightarrow}=H_{j}+\hbar^{2} \Delta_{s} H_{j}+O\left(\hbar^{4}\right) \quad(j=1, \ldots, n) .
$$

Here the quantum diffusion operator $\Delta_{s}$ is given by

$$
\Delta_{s} \stackrel{\text { def }}{\rightarrow}=\frac{1}{16} D^{2} s_{l} \cdot J \otimes J \cdot D^{2} s_{k} \cdot \frac{\partial^{2}}{\partial s_{l} \partial s_{k}}+\frac{1}{24} D^{2} s_{l} \cdot J \otimes J \cdot\left(D s_{k} \otimes D s_{m}\right) \frac{\partial^{3}}{\partial s_{l} \partial s_{k} \partial s_{m}}
$$

(the summation over repeated Latin indices ranges from 1 to $n$ ). This is the very operator entering the general quantum composite function expansion,

$$
k(\widehat{S})=\left(I-\hbar^{2} \Delta_{S}+O\left(\hbar^{4}\right)\right) k(S),
$$

where the set of operators $\widehat{S}=\left(\widehat{S}_{1}, \ldots, \widehat{S}_{n}\right)$ on the left is assumed to be symmetrized (Weyl ordered), see, e.g., [13, Appendix 1, formula (1.37)].

Theorem 2.1. Let $\left\{\widehat{H}_{j}\right\}$ be a quantum integrable system, and let the energy functions $H_{j}^{\hbar}$ be defined by (2.7). Then the deformed tori $\left\{H^{\hbar}=\mathrm{const}\right\}$ annihilate the quantum symplectic form $\omega^{\hbar}(2.4)$ up to $O\left(\hbar^{4}\right)$. Equivalently, the energy functions $H_{j}^{\hbar}$ are in involution up to $O\left(\hbar^{4}\right)$ with respect to the bracket determined by the quantum Poisson tensor $\left(\omega^{\hbar}\right)^{-1}$.

Sketch of the proof. Let us write out the Jacobi identity for double commutators of the operator triple $\widehat{X^{\alpha}}, \widehat{X^{\beta}}, \widehat{X^{\gamma}}$ with the vector function $X$ defined by (2.3). Taking into account formula (2.6) and using the relation $\{X \stackrel{\otimes}{\rightarrow}, X\}=-J=$ const, we obtain

$$
\underset{\alpha, \beta, \gamma}{\rightarrow} \mathfrak{S}\left\{X^{\alpha},\left\langle\left\langle X^{\beta}, X^{\gamma}\right\rangle\right\rangle\right\}=0 .
$$

Here the symbol $\mathfrak{S}$ stands for cyclic summation. The Poisson bracket operation in (2.10) can be replaced by the derivation operation in the $X$-coordinates,

$$
\underset{\alpha, \beta, \gamma}{\rightarrow} \mathfrak{S} J^{\alpha \alpha^{\prime}}\left(\partial / \partial X^{\alpha^{\prime}}\right)\left\langle\left\langle X^{\beta}, X^{\gamma}\right\rangle\right\rangle=0,
$$

or, equivalently,

$$
\underset{\alpha, \beta, \gamma}{\rightarrow} \mathfrak{S}\left(\partial / \partial X^{\alpha}\right) J_{\beta \beta^{\prime}}\left\langle\left\langle X^{\beta^{\prime}}, X^{\gamma^{\prime}}\right\rangle\right\rangle J_{\gamma^{\prime} \gamma}=0
$$


(the summation over repeated Greek indices ranges from 1 to $2 n$ ). The last identity implies that the quantum form $\omega^{\hbar}(2.4)$ is closed up to $O\left(\hbar^{4}\right)$, and thus it is symplectic.

The quantum Poisson bracket is given by

$$
\{A, B\}^{\hbar} \stackrel{\text { def }}{\rightarrow}=d A\left(\omega^{\hbar}\right)^{-1} d B=\{A, B\}-\hbar^{2} \frac{\partial A}{\partial X^{\alpha}}\left\langle\left\langle X^{\alpha}, X^{\beta}\right\rangle\right\rangle \frac{\partial B}{\partial X^{\beta}}+O\left(\hbar^{4}\right) .
$$

Taking into account formula (2.1), we can derive the relation

$$
\left\{H_{j}, H_{k}\right\}=\hbar^{2}\left\langle\left\langle H_{j}^{0}, H_{k}^{0}\right\rangle\right\rangle+O\left(\hbar^{4}\right),
$$

and then it follows from (2.11) that

$\left\{H_{j}^{\hbar}, H_{k}^{\hbar}\right\}^{\hbar}=\hbar^{2}\left(\left\langle\left\langle H_{j}^{0}, H_{k}^{0}\right\rangle\right\rangle-\frac{\partial H_{j}^{0}}{\partial s_{l}}\left\langle\left\langle s_{l}, s_{m}\right\rangle\right\rangle \frac{\partial H_{k}^{0}}{\partial s_{m}}+\left\{H_{j}^{0}, \Delta_{s} H_{k}^{0}\right\}-\left\{H_{k}^{0}, \Delta_{s} H_{j}^{0}\right\}\right)+O\left(\hbar^{4}\right)$.

In Lemma 2.1 below, we prove that the coefficient at $\hbar^{2}$ on the right-hand side vanishes, i.e.,

$$
\left\{H_{j}^{\hbar}, H_{k}^{\hbar}\right\}^{\hbar}=O\left(\hbar^{4}\right)
$$

Lemma 2.1. For the classical integrable system $\left\{H_{j}^{0}\right\}$ with the action coordinates $\left\{s_{j}\right\}$, the following identities hold:

$$
\left\langle\left\langle H_{j}^{0}, H_{k}^{0}\right\rangle\right\rangle-\frac{\partial H_{j}^{0}}{\partial s_{l}}\left\langle\left\langle s_{l}, s_{m}\right\rangle\right\rangle \frac{\partial H_{k}^{0}}{\partial s_{m}}+\left\{H_{j}^{0}, \Delta_{s} H_{k}^{0}\right\}-\left\{H_{k}^{0}, \Delta_{s} H_{j}^{0}\right\}=0,
$$

where the quantum operations $\langle\langle\cdot, \cdot\rangle\rangle$ and $\Delta_{s}$ are determined by (2.5) and (2.8).

Proof. For the first two summands in (2.12), we can see from (2.5) and from the identities

$$
\left\{s_{m}, s_{i}\right\} \equiv-D s_{m} \cdot J \cdot D s_{i}=0
$$

that

$$
\begin{aligned}
\left\langle\left\langle H_{j}^{0},\right.\right. & \left.\left.H_{k}^{0}\right\rangle\right\rangle-\frac{\partial H_{j}^{0}}{\partial s_{l}}\left\langle\left\langle s_{l}, s_{m}\right\rangle\right\rangle \frac{\partial H_{k}^{0}}{\partial s_{m}} \\
& =\operatorname{Alt}_{j, k}\left\{\frac{\partial^{3} H_{k}^{0}}{\partial s_{l} \partial s_{m} \partial s_{r}}\left(D s_{l} \otimes D s_{m} \otimes D s_{r}\right) \cdot J \otimes J \otimes J \cdot D^{3} s_{i} \frac{\partial H_{j}^{0}}{\partial s_{i}}\right\} \\
& +3 \operatorname{Alt}_{j, k}\left\{\frac{\partial^{2} H_{k}^{0}}{\partial s_{l} \partial s_{m}}\left(D^{2} s_{l} \otimes D s_{m}\right) \cdot J \otimes J \otimes J \cdot D^{3} s_{i} \frac{\partial H_{j}^{0}}{\partial s_{i}}\right\} \\
& -\frac{\partial^{2} H_{j}^{0}}{\partial s_{l} \partial s_{m}} \mathfrak{S}\left(D^{2} s_{l} \otimes D s_{m}\right) \cdot J \otimes J \otimes J \cdot \mathfrak{S}\left(D^{2} s_{r} \otimes D s_{i}\right) \frac{\partial H_{k}^{0}}{\partial s_{r} \partial s_{i}} .
\end{aligned}
$$

Here the symbol Alt stands for the skew-symmetric summation

$$
\operatorname{Alt}_{j, k}\left\{A_{j k}\right\} \stackrel{\text { def }}{\rightarrow}=A_{j k}-A_{k j}
$$

and the symbol $\mathfrak{S}$ for the cyclic summation

$$
\mathfrak{S}\left(D^{2} \otimes D\right)_{\alpha \beta \gamma}=D_{\alpha \beta}^{2} \otimes D_{\gamma}+D_{\beta \gamma}^{2} \otimes D_{\alpha}+D_{\gamma \alpha}^{2} \otimes D_{\beta} .
$$


It can readily be seen that the last summand on the right-hand side of (2.13) vanishes.

For the third and fourth summands in (2.12), it follows from (2.8) that

$$
\begin{aligned}
\left\{H_{j}^{0},\right. & \left.\Delta_{s} H_{k}^{0}\right\}-\left\{H_{k}^{0}, \Delta_{s} H_{j}^{0}\right\} \\
& =\operatorname{Alt}_{j, k}\left\{\frac{\partial^{3} H_{k}^{0}}{\partial s_{l} \partial s_{m} \partial s_{r}} D_{\alpha^{\prime} \beta \gamma}^{2} s_{l} \cdot D_{\beta^{\prime}} s_{m} \cdot D_{\gamma^{\prime}} s_{r} \cdot J^{\alpha^{\prime} \alpha} J^{\beta^{\prime} \beta} J^{\gamma^{\prime} \gamma} D_{\alpha} s_{i} \frac{\partial H_{j}^{0}}{\partial s_{i}}\right\} \\
& +3 \operatorname{Alt}_{j, k}\left\{\frac{\partial^{2} H_{k}^{0}}{\partial s_{l} \partial s_{m}} D_{\alpha^{\prime} \beta^{\prime}}^{2} s_{l} \cdot D_{\alpha \beta \gamma^{\prime}}^{3} s_{m} J^{\alpha^{\prime} \alpha} J^{\beta^{\prime} \beta} J^{\gamma^{\prime} \gamma} D_{\gamma} s_{i} \frac{\partial H_{j}^{0}}{\partial s_{i}}\right\} \\
& +2 \operatorname{Alt}_{j, k}\left\{\frac{\partial^{3} H_{k}^{0}}{\partial s_{l} \partial s_{m} \partial s_{r}} D_{\beta \gamma}^{2} s_{l} \cdot D_{\alpha^{\prime} \beta^{\prime}}^{2} s_{r} \cdot D_{\gamma^{\prime}}^{2} s_{m} \cdot J^{\alpha^{\prime} \alpha} J^{\beta^{\prime} \beta} J^{\gamma^{\prime} \gamma} D_{\alpha} s_{i} \frac{\partial H_{j}^{0}}{\partial s_{i}}\right\} .
\end{aligned}
$$

The second summands (with the coefficients 3) on the right-hand sides of (2.13) and (2.14) are combined together into the expression

$$
\begin{aligned}
3 \operatorname{Alt}_{j, k} & \left\{\frac{\partial^{2} H_{k}^{0}}{\partial s_{l} \partial s_{m}} D_{\alpha^{\prime} \beta^{\prime}}^{2} s_{l}\left(D_{\gamma^{\prime}} s_{m} D_{\alpha \beta}^{2}\left(D_{\gamma} s_{i}\right)+D_{\alpha \beta}^{2}\left(D_{\gamma^{\prime}} s_{m}\right) \cdot D_{\gamma} s_{i}\right) \cdot J^{\alpha^{\prime} \alpha} J^{\beta^{\prime} \beta} J^{\gamma^{\prime} \gamma} \frac{\partial H_{j}^{0}}{\partial s_{i}}\right\} \\
= & -3 \operatorname{Alt}_{j, k}\left\{\frac{\partial^{2} H_{k}^{0}}{\partial s_{l} \partial s_{m}} D_{\alpha^{\prime} \beta^{\prime}}^{2} s_{l} D_{\alpha \beta}^{2}\left\{s_{m}, s_{i}\right\} \cdot J^{\alpha^{\prime} \alpha} J^{\beta^{\prime} \beta} \frac{\partial H_{j}^{0}}{\partial s_{i}}\right\} \\
& -3 \operatorname{Alt}_{j, k}\left\{\frac{\partial^{2} H_{k}^{0}}{\partial s_{l} \partial s_{m}} D_{\alpha^{\prime} \beta^{\prime}}^{2} s_{l}\left(D_{\alpha \gamma^{\prime}}^{2} s_{m} \cdot D_{\beta \gamma}^{2} s_{i}+D_{\beta \gamma^{\prime}}^{2} s_{m} \cdot D_{\alpha \gamma}^{2} s_{i}\right) \cdot J^{\alpha^{\prime} \alpha} J^{\beta^{\prime} \beta} J^{\gamma^{\prime} \gamma} \frac{\partial H_{j}^{0}}{\partial s_{i}}\right\} .
\end{aligned}
$$

The last term here vanishes, as well as the bracket $\left\{s_{m}, s_{i}\right\}=0$. Thus, the second summands in (2.13) and (2.14) taken together give the zero value.

Thus, taking (2.13) and (2.14) together, we obtain

$$
\begin{gathered}
\left\langle\left\langle H_{j}^{0}, H_{k}^{0}\right\rangle\right\rangle-\frac{\partial H_{j}^{0}}{\partial s_{l}}\left\langle\left\langle s_{l}, s_{m}\right\rangle\right\rangle \frac{\partial H_{k}^{0}}{\partial s_{m}}+\left\{H_{j}^{0}, \Delta_{s} H_{k}^{0}\right\}-\left\{H_{k}^{0}, \Delta_{s} H_{j}^{0}\right\} \\
=\operatorname{Alt}_{j, k}\left\{\frac { \partial ^ { 3 } H _ { k } ^ { 0 } } { \partial s _ { l } \partial s _ { m } \partial s _ { r } } \left(D_{\alpha^{\prime}} s_{l} \cdot D_{\beta^{\prime}} s_{m} \cdot D_{\gamma^{\prime}} s_{r} \cdot D_{\alpha \beta \gamma}^{3} s_{i}+D_{\beta \gamma \alpha^{\prime}}^{3} s_{l} \cdot D_{\beta^{\prime}} s_{m} \cdot D_{\gamma^{\prime}} s_{r} \cdot D_{\alpha} s_{i}\right.\right. \\
\left.\left.\quad+2 D_{\beta \alpha^{\prime}}^{2} s_{l} \cdot D_{\beta^{\prime}} s_{m} \cdot D_{\gamma^{\prime}} s_{r} \cdot D_{\gamma \alpha}^{2} s_{i}\right) J^{\alpha^{\prime} \alpha} J^{\beta^{\prime} \beta} J^{\gamma^{\prime} \gamma} \frac{\partial H_{j}^{0}}{\partial s_{i}}\right\} \\
-2 \operatorname{Alt}_{j, k}\left\{\frac { \partial ^ { 3 } H _ { k } ^ { 0 } } { \partial s _ { l } \partial s _ { m } \partial s _ { r } } \left(D_{\beta \alpha^{\prime}}^{2} s_{l} \cdot D_{\beta^{\prime}} s_{m} \cdot D_{\gamma^{\prime}} s_{r} \cdot D_{\gamma \alpha}^{2} s_{i}\right.\right. \\
\left.\left.\quad-D_{\beta \gamma}^{2} s_{l} \cdot D_{\alpha^{\prime} \beta^{\prime}}^{2} s_{r} \cdot D_{\gamma^{\prime}} s_{m} \cdot D_{\alpha} s_{i}\right) J^{\alpha^{\prime} \alpha} J^{\beta^{\prime} \beta} J^{\gamma^{\prime} \gamma} \frac{\partial H_{j}^{0}}{\partial s_{i}}\right\} .
\end{gathered}
$$

The last term of $\mathrm{Alt}_{j, k}$ on the right-hand side of (2.15) (with the coefficient 2) vanishes, and the first term of $\mathrm{Alt}_{j, k}$ gives the expression

$$
-\operatorname{Alt}_{j, k}\left\{\frac{\partial^{3} H_{k}^{0}}{\partial s_{l} \partial s_{m} \partial s_{r}} D_{\beta \gamma}^{2}\left\{s_{l}, s_{i}\right\} J^{\beta^{\prime} \beta} J^{\gamma^{\prime} \gamma} D_{\beta^{\prime}} s_{m} \cdot D_{\gamma^{\prime}} s_{r} \frac{\partial H_{j}^{0}}{\partial s_{i}}\right\} .
$$

Since $\left\{s_{l}, s_{i}\right\}=0$, we conclude that this expression, and thus the entire expression (2.15), is identically zero. 
Remark 2.1. Let us pay attention to the following important fact. The quantum Poisson bracket (2.11), as well as the quantum symplectic form (2.4) and the energy functions (2.7), contains not only the first derivative but also the second and the third derivatives of phase space coordinates and Hamiltonians. Actually, the higher-order $\hbar$-corrections in (2.4), (2.7), and (2.11) contain higher and higher derivatives (see Section 4). Objects of this kind, depending on higher derivatives, look unusual from the viewpoint of classical differential geometry. This is the very distinction characterizing quantum geometry. The presence of higher derivatives reflects the phenomena of quantum diffusion and uncertainty. In spite of these awkward objects, the quantum geometry is indeed a geometry, thanks to identities like (2.12), where the quantum diffusion and dispersion operations $\Delta_{s}$ and $\langle\langle\cdot, \cdot\rangle\rangle$ correlate with each other and with the classical Poisson bracket.

\section{Spectrum and dynamics using quantum actions-angles}

By Theorem 2.1, the set of energy functions $\left\{H_{j}^{\hbar}\right\}$ determines $\bmod O\left(\hbar^{4}\right)$ a classical integrable system with respect to the quantum symplectic structure $\omega^{\hbar}(2.4)$. The deformed tori $\left\{H^{\hbar}=\right.$ const $\}$ described in Theorem 2.1 fiber a domain in the phase space. Hence, in this domain, with accuracy up to $O\left(\hbar^{4}\right)$, one can construct the action-angle coordinates $\left(s^{\hbar}, \tau^{\hbar}\right), 0 \leq \tau^{\hbar j} \leq 2 \pi$, in the standard way. We refer to these coordinates as the quantum action-angles. The quantum actions are constant along the deformed tori. The explicit formula for the quantum actions is

$$
s_{j}^{\hbar}=\frac{1}{2 \pi} \int_{C_{j}^{\hbar}} \alpha^{\hbar}, \quad d \alpha^{\hbar}=\omega^{\hbar} .
$$

Here $C_{j}^{\hbar}$ is the $j$ th basic cycle on the deformed torus $\left\{H^{\hbar}=\right.$ const $\}$ containing the given phase-space point. Let us choose an $\hbar$-equidistant grid of deformed tori by taking discrete values of the quantum actions as follows:

$$
s^{\hbar}=\mu+N \hbar, \quad N \in \mathbb{Z}^{n}, \quad N \sim \hbar^{-1} .
$$

Here $\mu=\left(\mu_{1}, \ldots, \mu_{m}\right)$ is a constant vector with the components

$$
\mu_{j}=\frac{\hbar}{4} m_{j}+O\left(\hbar^{2}\right), \quad j=1, \ldots, n,
$$

where $m_{j}$ are the Maslov indices of the basic cycles on the tori.

Theorem 3.1. Let $H_{j}^{\hbar}(j=1, \ldots, n)$ be the energy functions (2.7) of a quantum integrable system $\left\{\widehat{H}_{j}\right\}(2.1)$, and let $s_{j}^{\hbar}$ be the quantum actions (3.0) determined by the deformed integrable system $\left\{H_{j}^{\hbar}\right\}$ described in Theorem 2.1. In this case, for a certain vector $\mu$ of type (3.2), the sequence of numbers

$$
E_{j}[N] \stackrel{\text { def }}{\rightarrow}=\left.H_{j}^{\hbar}\right|_{s^{\hbar}=\mu+\hbar N}, \quad N \in \mathbb{Z}^{n},
$$

approximates the eigenvalues of the operator $\widehat{H}_{j}$ up to $O\left(\hbar^{4}\right)$, i.e.,

$$
\operatorname{dist}\left(E_{j}[N], \operatorname{Spectr} \widehat{H}_{j}\right)=O\left(\hbar^{4}\right) \text {. }
$$


Sketch of the proof. The quantum symplectic form (2.4) reads

$\omega^{\hbar}=d s_{j} \wedge d \tau^{j}+\frac{\hbar^{2}}{2}\left\langle\left\langle s_{l}, s_{j}\right\rangle\right\rangle d \tau^{l} \wedge d \tau^{j}+\frac{\hbar^{2}}{2}\left\langle\left\langle\tau^{l}, \tau^{j}\right\rangle\right\rangle d s_{l} \wedge d s_{j}+\hbar^{2}\left\langle\left\langle s_{j}, \tau^{l}\right\rangle\right\rangle d s_{l} \wedge d \tau^{j}+O\left(\hbar^{4}\right)$.

On the other hand, by the definition of the quantum action-angle coordinates, we have $\omega^{\hbar}=d s^{\hbar} \wedge d \tau^{\hbar}$. Let us substitute into (3.4) the $\hbar$-expansions for the quantum action-angle coordinates, i.e.,

$$
s^{\hbar}=s+\hbar^{2} a+O\left(\hbar^{4}\right), \quad \tau^{\hbar}=\tau+\hbar^{2} \phi+O\left(\hbar^{4}\right) .
$$

Then it follows from (3.4) that the coefficients $a$ and $\phi$ of expansion (3.5) satisfy the identities

$$
\frac{\partial a_{j}}{\partial \tau^{l}}-\frac{\partial a_{l}}{\partial \tau^{j}}=\left\langle\left\langle s_{l}, s_{j}\right\rangle\right\rangle, \quad \frac{\partial \phi^{j}}{\partial s_{l}}-\frac{\partial \phi^{l}}{\partial s_{j}}=\left\langle\left\langle\tau^{j}, \tau^{l}\right\rangle\right\rangle, \quad \frac{\partial a_{j}}{\partial s_{l}}+\frac{\partial \phi^{l}}{\partial \tau^{j}}=\left\langle\left\langle s_{j}, \tau^{l}\right\rangle\right\rangle .
$$

In view of (2.6), this system implies the commutation relations

$$
\frac{i}{\hbar}\left[\widehat{s_{j}^{\hbar}}, \widehat{s_{l}^{\hbar}}\right]=O\left(\hbar^{4}\right), \quad \frac{i}{\hbar}\left[\widehat{\tau^{\hbar j}}, \widehat{\tau^{\hbar} l}\right]=O\left(\hbar^{4}\right), \quad \frac{i}{\hbar}\left[\widehat{s_{j}^{\hbar}}, \widehat{\tau^{\hbar} l}\right]=\delta_{j}^{l}+O\left(\hbar^{4}\right)
$$

Denote by $\operatorname{ad}_{\widehat{A}}$ the commutator operation $\operatorname{ad}_{\widehat{A}}(\widehat{B}) \stackrel{\text { def }}{\rightarrow}=[\widehat{A}, \widehat{B}]$. It follows from (3.6) that, for each $j, j=1, \ldots, n$, the exponential function generated by $\frac{i}{\hbar} \operatorname{ad}_{\widehat{s_{j}^{\hbar}}}$ is $2 \pi$-periodic $\bmod O\left(\hbar^{4}\right)$, i.e.,

$$
\exp \left\{\frac{2 \pi i}{\hbar} \operatorname{ad}_{\widehat{s_{j}^{\hbar}}}\right\}=I+O\left(\hbar^{4}\right) .
$$

Since the algebra representation $A \rightarrow \widehat{A}$ is irreducible, it follows from (3.7) that the exponential function generated by the operator $\frac{i}{\hbar} \widehat{s_{j}^{\hbar}}$ is $2 \pi$-periodic up to $\bmod O\left(\hbar^{4}\right)$ and up to a unitary constant multiplier

$$
\exp \left\{\frac{2 \pi i}{\hbar} \widehat{s_{j}^{\hbar}}\right\}=\exp \left\{\frac{2 \pi i}{\hbar} \mu_{j}\right\} \cdot I+O\left(\hbar^{4}\right), \quad \mu_{j}=\text { const } .
$$

The leading part of the Hamiltonian $s_{j}^{\hbar}$ is the classical action $s_{j}$ (see (3.5)). The Hamiltonian flow generated by $s_{j}$ is $2 \pi$-periodic. As is known [14, 15], under this assumption, the constant $\mu_{j}$ in (3.8) must be of the form (3.2).

It follows from (3.8) that the spectrum of $\widehat{s_{j}^{\hbar}}$ contains the sequence (3.1) (up to $\left.O\left(\hbar^{4}\right)\right)$.

Note that the energy function $H_{j}^{\hbar}$ depends on the quantum action coordinates only, i.e., there exists a function $f_{j}^{\hbar}$ in $n$ variables for which

$$
H_{j}^{\hbar}=f_{j}^{\hbar}\left(s^{\hbar}\right)
$$

It follows from (2.9) and (3.9) that

$$
f_{j}^{\hbar}\left(\widehat{s^{\hbar}}\right)=\widehat{H_{j}^{\hbar}}-\hbar^{2} \widehat{\Delta_{s^{\hbar}} H_{j}^{\hbar}}+O\left(\hbar^{4}\right) .
$$


By (2.7), we have

$$
\widehat{H}_{j}=f_{j}^{\hbar}\left(\widehat{s^{\hbar}}\right)+O\left(\hbar^{4}\right) .
$$

By substituting the approximate eigenvalues (3.1) of the commuting $\bmod O\left(\hbar^{5}\right)$ operators $\widehat{s^{\hbar}}$ into formula (3.10), we conclude that the spectrum of $\widehat{H}_{j}$ contains the sequence

$$
f_{j}^{\hbar}(\mu+\hbar N)+O\left(\hbar^{4}\right)
$$

In view of (3.9), this sequence coincides with $E_{j}[N]+O\left(\hbar^{4}\right)$.

Remark 3.1. The idea to compute the semiclassical spectrum using quantum action coordinates was suggested in [16] (for details, see [9]). The new feature in Theorem 3.1 is the explicit geometric formula (3.0) for the quantum actions in terms of the quantum symplectic structure and the deformed tori. Applying this formula, one can readily compute, for instance, the first quantum corrections to classical actions in expansion (3.5), namely,

$$
a_{j}=\left(\frac{\partial H^{0}}{\partial s}\right)_{j}^{-1 l}\left(L_{l}-\left\langle L_{l}\right\rangle_{j}\right)+(1 /(2 \pi)) \int_{\Sigma_{j}^{0}} \varkappa+a_{j}^{0} .
$$

Here $a_{j}^{0}$ are some constants. In (3.11) we also use the notation

$$
L_{l} \stackrel{\text { def }}{\rightarrow}=\Delta_{s} H_{l}^{0}+M_{l},
$$

where the symbols $M_{l}$ stand for the $\hbar^{2}$-corrections in the expansion of original Hamiltonians of the quantum integrable system,

$$
H_{l}=H_{l}^{0}+\hbar^{2} M_{l}+\cdots .
$$

The angular brackets \langle\rangle$_{j}$ in (3.11) stand for the average over the classical angle $0 \leq$ $\tau^{j} \leq 2 \pi$. The membrane (i.e., spanning surface) $\Sigma_{j}^{0}$ in (3.11) is bounded by the $j$ th basic cycle on the classical torus $\left\{H^{0}=\right.$ const $\}$ containing the given phase space point. The form $\varkappa$ in (3.11) represents the $\hbar^{2}$-correction to the classical form in (3.4), i.e.,

$$
\varkappa=\frac{1}{2}\left\langle\left\langle s_{l}, s_{j}\right\rangle\right\rangle d \tau^{l} \wedge d \tau^{j}+\frac{1}{2}\left\langle\left\langle\tau^{l}, \tau^{j}\right\rangle\right\rangle d s_{l} \wedge d s_{j}+\left\langle\left\langle s_{j}, \tau^{l}\right\rangle\right\rangle d s_{l} \wedge d \tau^{j} .
$$

Remark 3.2. In view of (3.0), the quantization rule (3.3) can be represented as a system of equations for the energy levels $E=E[N]$, namely,

$$
\frac{1}{2 \pi} \int_{C_{j}^{\hbar}[E]} \alpha^{\hbar}=\mu_{j}+\hbar N_{j} \quad(j=1, \ldots, n) .
$$

Here $E=\left(E_{1}, \ldots, E_{n}\right)$ are the energy levels sought for, i.e., the eigenvalues of the commuting Hamiltonians $\widehat{H}_{j}$. The basic cycles $C_{j}^{\hbar}[E]$ belong to the deformed torus $\left\{H^{\hbar}=E\right\}$, where $H_{j}^{\hbar}$ are the energy functions (2.7) assigned to $H_{j}$. The constants $\mu_{j}$ in (3.3), (3.12) are determined using the holonomy generated by the quantum actions following formulas (3.8). The leading term in expansion (3.2) for the vector $\mu$ is presented by the Maslov indices of the basic cycles on the tori, and the higher $\hbar$-terms determine certain quantum corrections. 
Now let us note that, by using the representation (3.10) and the commutation relations (3.6), the quantum dynamics generated by each Hamiltonian $\widehat{H}_{j}$ can readily be computed as follows:

$$
\exp \left\{(i t / \hbar) \operatorname{ad}_{\widehat{H}_{j}}\right\} g^{[0]}\left(\stackrel{2}{\rightarrow} \widehat{\tau^{\hbar}}, \stackrel{1}{\rightarrow} \widehat{s^{\hbar}}\right)=g_{j}^{[t]}\left(\stackrel{2}{\rightarrow} \widehat{\tau^{\hbar}}, \stackrel{1}{\rightarrow} \widehat{s^{\hbar}}\right)
$$

Here the function $g^{[0]}$ represents the initial quantum observable or quantum density, and $g_{j}^{[t]}$ represents its time evolution, i.e.,

$$
g_{j}^{[t]}\left(\tau^{\hbar}, s^{\hbar}\right)=\exp \left\{(i t / \hbar)\left(f_{j}^{\hbar}\left(s^{\hbar}-i \hbar\left(\partial / \partial \tau^{\hbar}\right)\right)-f_{j}^{\hbar}\left(s^{\hbar}\right)\right)+O\left(t \hbar^{4}\right)\right\} g^{[0]}\left(\tau^{\hbar}, s^{\hbar}\right) .
$$

It follows from this formula that the quantum action coordinates are constant provided that $t \hbar^{4} \ll 1$, and all the dynamics is developed in quantum angle coordinates only.

Theorem 3.2. The deformed tori $\left\{H^{\hbar}=\right.$ const $\}$ are preserved by the quantum integrable dynamics in the long-time range $t \sim o\left(\hbar^{-4}\right)$. Along these tori, the dynamics generated by $\widehat{H}_{j}$ looks like a multiperiodic rotation in the quantum angle coordinates $\tau^{\hbar}$ with the frequencies $\partial H_{j}^{\hbar} / \partial s^{\hbar}$ as long as $t \sim o\left(\hbar^{-1}\right)$. At the time frontier $t \sim \hbar^{-1}$, the longitudinal dynamics diffuses to the entire torus and, as long as $t \sim o\left(\hbar^{-4}\right)$, this dynamics is given by formulas (3.13) and (3.14).

Note that by some transformation of the phase space near the identity, the quantum symplectic form $\omega^{\hbar}(2.4)$ can be turned back to the classical form $\omega$ (2.3); however, the Hamiltonian (or the energy) functions $H_{j}^{\hbar}$ of the deformed integrable system do not come back to $H_{j}^{0}$ under this transformation. Thus, the Hamiltonian dynamics on deformed tori from Theorem 3.2 is not isomorphic to the original Hamiltonian dynamics on the classical tori.

Besides the Hamiltonian multiperiodic rotation, Theorem 3.2 describes the character of quantum spreading in the long time range. We see that the spreading does not take place at all in directions transversal to the deformed tori. In particular, one can claim that there is no quantum chaos for the integrable system until $t \sim o\left(\hbar^{-4}\right)$.

Along the deformed tori, the quantum spreading is explicitly determined by formula (3.14). The leading diffusion process in (3.14)

$$
\exp \left\{-i \frac{t \hbar}{2} \mathcal{D}_{j} \frac{\partial^{2}}{\partial \tau^{\hbar} \partial \tau^{\hbar}}\right\}, \quad t \sim \hbar^{-1}, \quad \mathcal{D}_{j} \stackrel{\text { def }}{\rightarrow}=\frac{\partial^{2} H_{j}^{\hbar}}{\partial s^{\hbar} \partial s^{\hbar}},
$$

is easily computed via the theta function in the quantum angle coordinates globally over each deformed torus

$$
\left\{s^{\hbar}=\mu+\hbar N, \quad 0 \leq \tau^{\hbar l} \leq 2 \pi \quad(l=1, \ldots, n)\right\} .
$$

Remark 3.3. The quantum angle coordinates are obtained geometrically from the representation $\omega^{\hbar}=d s^{\hbar} \wedge d \tau^{\hbar}$ of the quantum symplectic structure. The explicit expression for the first quantum corrections to the classical angles in expansion (3.5) is the following:

$$
\phi^{l}=\int_{0}^{\tau}\left(\left\langle\left\langle s_{j}, \tau^{l}\right\rangle\right\rangle-\frac{\partial a_{j}}{\partial s_{l}}\right) d \tau^{j}+\varphi^{l}(s)
$$


The integration in (3.15) is taken along any path in $\tau$-space, $a_{j}$ are the first quantum corrections to the classical actions given by (3.11) and $\varphi^{l}$ are obtained from the initial condition at $\tau=0$ :

$$
\frac{\partial \varphi^{l}}{\partial s_{j}}-\frac{\partial \varphi^{j}}{\partial s_{l}}=\left.\left\langle\left\langle\tau^{l}, \tau^{j}\right\rangle\right\rangle\right|_{\tau=0}
$$

The right-hand side in this condition presents coefficients of a closed form in $s$-space (see $(2.10))$ :

$$
\underset{k, l, j}{\rightarrow} \mathfrak{S} \frac{\partial}{\partial s_{k}}\left\langle\left\langle\tau^{l}, \tau^{j}\right\rangle\right\rangle=0
$$

Thus, by the Poincaré lemma, one can easily resolve this initial condition as follows

$$
\varphi^{l}(s)=\left.\int_{0}^{1}\left\langle\left\langle\tau^{l}, \tau^{j}\right\rangle\right\rangle\right|_{\tau=0}\left(s \xi+s^{0}(1-\xi)\right)\left(s-s^{0}\right)_{j} \xi d \xi+\frac{\partial \psi(s)}{\partial s_{l}},
$$

where $s^{0}$ is some chosen point in the $s$-space and $\psi$ is an arbitrary function in $s$ coordinates. Formula (3.16) finally fixes the expression (3.15) for quantum corrections to the classical angle-coordinates up to a "gauge" freedom $\partial \psi(s) / \partial s_{l}$.

Note that (3.15) determines the correction $\phi^{l}$ as a $2 \pi$-periodic function in classical angles. It is guaranteed by the identities

$$
\left\langle\left\langle\left\langle s_{j}, \tau^{l}\right\rangle\right\rangle\right\rangle_{j}=\frac{\partial}{\partial s_{l}}\left\langle a_{j}\right\rangle_{j} \quad(j, l=1, \ldots, n) .
$$

To prove (3.17), let us note that (3.7), in the $\hbar^{2}$-term, implies

$$
\frac{\partial}{\partial s_{l}}\left\langle a_{j}\right\rangle_{j} \frac{\partial}{\partial \tau^{l}}-\frac{\partial}{\partial \tau^{l}}\left\langle a_{j}\right\rangle_{j} \frac{\partial}{\partial s_{l}}=\frac{1}{2 \pi} \int_{0}^{2 \pi} e^{\nu\left\{s_{j}, \cdot\right\}}\left\langle\left\langle s_{j}, \cdot\right\rangle\right\rangle e^{-\nu\left\{s_{j}, \cdot\right\}} d \nu .
$$

Since the left-hand side here is a first-order differential operator, the right-hand side has to be of the same type (in spite of the fact that $\left\langle\left\langle s_{j}, \cdot\right\rangle\right\rangle$ is a third-order operator, see (2.5)). Thus, all coefficients at the second and third derivatives on the right-hand side of (3.18) vanish, and we derive

$$
\frac{1}{2 \pi} \int_{0}^{2 \pi} e^{\nu\left\{s_{j}, \cdot\right\}}\left\langle\left\langle s_{j}, \cdot\right\rangle\right\rangle e^{-\nu\left\{s_{j}, \cdot\right\}} d \nu=\left\langle\left\langle\left\langle s_{j}, \tau^{l}\right\rangle\right\rangle\right\rangle_{j} \frac{\partial}{\partial \tau^{l}}+\left\langle\left\langle\left\langle s_{j}, s_{l}\right\rangle\right\rangle\right\rangle_{j} \frac{\partial}{\partial s_{l}} .
$$

Comparison with the left-hand side of (3.18) implies (3.17) and so the $2 \pi$-periodicity of (3.15).

\section{General algorithm for the equivalence of quantum and classical integrable systems}

Let the algebra of quantum observables be realized in a space of functions over a certain manifold $\mathfrak{X}$. The operator representation of this algebra

$$
A \rightarrow \widehat{A}
$$

is assumed to be Hermitian, invertible, and irreducible. We denote by $*$ the product operation:

$$
\widehat{A * B}=\widehat{A} \widehat{B}
$$


which has the properties $\overline{A * B}=\bar{B} * \bar{A}$ and $A * 1=A$.

The anticommutator $A \circledast B \stackrel{\text { def }}{\rightarrow}=\frac{1}{2}(A * B+B * A)$ is assumed to admit an expansion in even powers of the quantization parameter

$$
A \circledast B=A B-\hbar^{2} A \odot B, \quad \odot=\stackrel{(0)}{\rightarrow} \odot+\hbar^{2} \stackrel{(1)}{\rightarrow} \odot+\cdots .
$$

The leading term $A B$ is just the usual product of functions, and higher $\hbar$-terms in (4.2) are given by real symmetric bidifferential operations $\stackrel{(\alpha)}{\rightarrow} \odot$ of order $2 \alpha+2$.

The commutator $[A, B]_{*} \stackrel{\text { def }}{\rightarrow}=\frac{i}{\hbar}(A * B-B * A)$ is assumed to admit the $\hbar$-expansion:

$$
[A, B]_{*}=\{A, B\}-\hbar^{2}\left\langle\langle A, B\rangle, \quad\langle\langle,\rangle\rangle=\langle\langle,\rangle\rangle^{(0)}+\hbar^{2}\langle\langle,\rangle\rangle^{(1)}+\cdots,\right.
$$

whose leading term is the Poisson bracket operation

$$
\{A, B\}=d A \Psi d B
$$

and higher $\hbar$-terms are given by real skew-symmetric bidifferential operations $\langle\langle\cdot, \cdot\rangle\rangle^{(\alpha)}$ of order $2 \alpha+3$.

Since the representation (4.1) is irreducible, the Poisson tensor $\Psi$ (4.4) is invertible and the inverse tensor

$$
\omega=\Psi^{-1}
$$

determines the symplectic form on the manifold $\mathfrak{X}$. In particular, the dimension of $\mathfrak{X}$ is even: $\operatorname{dim} \mathfrak{X}=2 n$.

For a set of functions $S=\left(S_{1}, \ldots, S_{n}\right)$ on $\mathfrak{X}$ and a function $k$ of $n$ variables, one can define the symmetric (Weyl ordered) *-composite function $k(S)_{*}$, as well as the usual composite function $k(S)$. Their difference can be expanded into an $\hbar$-power series to $O\left(\hbar^{\infty}\right)$ :

$$
k(S)_{*}=\left(I-\hbar^{2} \Delta_{S}\right) k(S), \quad \Delta_{S}=\Delta_{S}^{(0)}+\hbar^{2} \Delta_{S}^{(1)}+\cdots
$$

The coefficients of expansion (4.6) are explicitly computed via the operations $\stackrel{(\alpha)}{\rightarrow} \odot$ from (4.2). For instance, the leading "diffusion" term in (4.6) reads

$$
\Delta_{S}^{(0)}=\frac{1}{2} S_{j} \stackrel{(0)}{\rightarrow} \odot S_{l} \frac{\partial^{2}}{\partial S_{j} \partial S_{l}}+\frac{1}{6}\left[\left[S_{j} \stackrel{(0)}{\rightarrow} \odot, S_{l}\right], S_{m}\right] \frac{\partial^{3}}{\partial S_{j} \partial S_{l} \partial S_{m}}
$$

Let $H_{1}, \ldots, H_{n}$ be the Hamiltonians of a quantum integrable system. Operators $\widehat{H}_{j}$ mutually commute, and so the functions $H_{j}$ commute with respect to the $*$-product:

$$
\left[H_{j}, H_{k}\right]_{*}=0 \text {. }
$$

Let this set of functions admit the $\hbar$-expansion to $O\left(\hbar^{\infty}\right)$ :

$$
H_{j}=H_{j}^{0}+\hbar^{2} M_{j}, \quad M_{j}=M_{j}^{(0)}+\hbar^{2} M_{j}^{(1)}+\cdots .
$$

The leading term of (4.9) determines the classical integrable system, i.e., the functions in involution with respect to the Poisson bracket (4.4). We assume that the level surfaces of these functions are compact. In a regular domain, where all $H_{j}^{0}$ are independent, the dimension of these surfaces (tori) is $n=\frac{1}{2} \operatorname{dim} \mathfrak{X}$. 
Denote by $s_{1}, \ldots, s_{n}$ classical action coordinates on this regular domain, and let $\tau^{1}, \ldots, \tau^{n}$ be the corresponding angle coordinated so that $H_{j}^{0}=f_{j}(s)$ and

$$
\left\{s_{j}, s_{l}\right\}=0, \quad\left\{\tau^{j}, \tau^{l}\right\}=0, \quad\left\{s_{j}, \tau^{l}\right\}=\delta_{j}^{l}
$$

In order to set up the equivalence of the quantum integrable system $\left\{\widehat{H}_{j}\right\}$ to a classical (deformed) integrable system, we first introduce the main notation and then explain how the algorithm of equivalence works.

The quantum action-angle coordinates $s^{\hbar}, \tau^{\hbar}$ are determined by $\hbar$-expansions to $O\left(\hbar^{\infty}\right)$

$$
\begin{array}{rlrl}
s^{\hbar} & =s+\hbar^{2} a, & & a=a^{(0)}+\hbar^{2} a^{(1)}+\cdots, \\
\tau^{\hbar}=\tau+\hbar^{2} \phi, & & \phi=\phi^{(0)}+\hbar^{2} \phi^{(1)}+\cdots,
\end{array}
$$

to obey the commutation relations

$$
\left[s_{j}^{\hbar}, s_{l}^{\hbar}\right]_{*}=O\left(\hbar^{\infty}\right), \quad\left[\tau^{\hbar j}, \tau^{\hbar l}\right]_{*}=O\left(\hbar^{\infty}\right), \quad\left[s_{j}^{\hbar}, \tau^{\hbar l}\right]_{*}=\delta_{j}^{l}+O\left(\hbar^{\infty}\right) .
$$

The choice of quantum actions must be consistent with the given quantum integrable system (4.8). Namely, the Hamiltonians $H_{j}$ must be expressed as $*$-composite functions in quantum actions to $O\left(\hbar^{\infty}\right)$ :

$$
H_{j}=f_{j}^{\hbar}\left(s^{\hbar}\right)_{*}, \quad f_{j}^{\hbar}=f_{j}+\hbar^{2} g_{j}, \quad g_{j}=g_{j}^{(0)}+\hbar^{2} g_{j}^{(1)}+\cdots .
$$

We associate each Hamiltonian $H_{j}$ with the energy function

$$
H_{j}^{\hbar} \stackrel{\text { def }}{\rightarrow}=f_{j}^{\hbar}\left(s^{\hbar}\right), \quad H_{j}^{\hbar}=H_{j}^{0}+\hbar^{2} L_{j}, \quad L_{j}=L_{j}^{(0)}+\hbar^{2} L_{j}^{(1)}+\cdots .
$$

In view of formula (4.6), we have the following relation:

$$
H_{j}=\left(I-\hbar^{2} \Delta_{s^{\hbar}}\right) H_{j}^{\hbar}
$$

and hence

$$
H_{j}^{\hbar}=\left(I-\hbar^{2} \Delta_{s^{\hbar}}\right)^{-1} H_{j}=\left(I+\sum_{k \geq 1} \hbar^{2 k}\left(\Delta_{s^{\hbar}}\right)^{k}\right) H_{j} .
$$

And finally, by means of the quantum action-angle coordinates, we define the quantum symplectic form

$$
\omega^{\hbar}=d s^{\hbar} \wedge d \tau^{\hbar}, \quad \omega^{\hbar}=\omega+\hbar^{2} \varkappa, \quad \varkappa=\varkappa^{(0)}+\hbar^{2} \varkappa^{(1)}+\cdots
$$

as well as the quantum Poisson tensor $\Psi^{\hbar}=\left(\omega^{\hbar}\right)^{-1}$ and the corresponding quantum bracket $\{A, B\}^{\hbar}=d A \Psi^{\hbar} d B$.

In order to construct all these quantum geometric objects, we proceed by induction.

At the zero induction step, the coefficients $a^{(0)}, \phi^{(0)}, g_{j}^{(0)}, L_{j}^{(0)}, \varkappa^{(0)}$ of $\hbar$-expansions (4.11), (4.13), (4.14), (4.16) are known from the procedure described in Section 2. Assume that we know all coefficients $a^{(\alpha)}, \phi^{(\alpha)}, g_{j}^{(\alpha)}, L_{j}^{(\alpha)}, \varkappa^{(\alpha)}$ for $\alpha \leq k-1$, and let us demonstrate how to compute them for $\alpha=k$.

The coefficients $L_{j}^{(k)}$ are computed by (4.15) using expansions (4.9). Thus the deformed tori $\left\{H^{\hbar}=\right.$ const $\}$ are known at the order $\hbar^{2 k}$. 
The form $\varkappa^{(k)}$ in (4.16) is given by

$$
\begin{aligned}
\varkappa^{(k)}=\frac{1}{2}\left(\frac{\partial a_{j}^{(k)}}{\partial \tau^{l}}\right. & \left.-\frac{\partial a_{l}^{(k)}}{\partial \tau^{j}}\right) d \tau^{l} \wedge d \tau^{j}-\frac{1}{2}\left(\frac{\partial \phi^{(k) j}}{\partial s_{l}}-\frac{\partial \phi^{(k) l}}{\partial s_{j}}\right) d s^{l} \wedge d s^{j} \\
& +\left(\frac{\partial a_{l}^{(k)}}{\partial s_{j}}+\frac{\partial \phi^{(k) j}}{\partial \tau^{l}}\right) d s_{j} \wedge d \tau^{l}+\sum_{0 \leq m \leq k-1} d a_{j}^{(m)} \wedge d \phi^{(k-m-1) j} .
\end{aligned}
$$

The combinations of first derivatives of functions $a^{(k)}$ and $\phi^{(k)}$ standing in first three summands are derived from commutation relations (4.12) at the order $\hbar^{2 k}$. For instance,

$$
\begin{aligned}
\frac{\partial a_{j}^{(k)}}{\partial \tau^{l}}-\frac{\partial a_{l}^{(k)}}{\partial \tau^{j}}=\langle\langle & \left.\left.s_{l}, s_{j}\right\rangle\right\rangle^{(k)}+\sum_{0 \leq m \leq k-1}\left\{a_{j}^{(m)}, a_{l}^{(k-m-1)}\right\} \\
& +\sum_{0 \leq m \leq k-1}\left(\left\langle\left\langle s_{l}, a_{j}^{(k-m-1)}\right\rangle\right\rangle^{(m)}-\left\langle\left\langle s_{j}, a_{l}^{(k-m-1)}\right\rangle\right\rangle^{(m)}\right) \\
& +\sum_{0 \leq m \leq k-2} \sum_{0 \leq r \leq k-m-2}\left\langle\left\langle a_{l}^{(r)}, a_{j}^{(k-m-r-2)}\right\rangle\right\rangle^{(m)} .
\end{aligned}
$$

Analogous equations hold for $\partial \phi^{(k) j} / \partial s_{l}-\partial \phi^{(k) l} / \partial s_{j}$ and for $\partial a_{l}^{(k)} / \partial s_{j}+\partial \phi^{(k) j} / \partial \tau^{l}$. The right-hand sides of these equations are known by the inductive hypothesis. By substituting these right-hand sides into the first three summands in (4.17), we derive a formula for $\varkappa^{(k)}$ via $a^{(\alpha)}$ and $\phi^{(\alpha)}$ with $\alpha \leq k-1$. Thus we compute the quantum form $\omega^{\hbar}(4.16)$ at the order $\hbar^{2 k}$.

Then it has to be checked that the form $\omega^{\hbar}$ vanishes on the deformed tori up to $O\left(\hbar^{2 k+2}\right)$.

As soon as we know a symplectic structure and a tori fibration annihilating the symplectic form up to $O\left(\hbar^{2 k+2}\right)$, the action-angle coordinates are automatically constructed with the same accuracy. Therefore, we have obtained $a^{(k)}$ and $\phi^{(k)}$ as well as the coefficient $g^{(k)}$ via expansions (4.14), (4.13). The induction is completed.

Proposition. Theorems 2.1, 3.1, and 3.2 hold with accuracy $O\left(\hbar^{\infty}\right)$ (instead of $O\left(\hbar^{4}\right)$ ) and at the time range $t \sim O\left(\hbar^{-\infty}\right)$ (instead of $O\left(\hbar^{-4}\right)$ ) in the general setting described in the present section.

It is important to stress that the algorithm for $\hbar^{\infty}$-equivalence of quantum and classical integrability described at this section works in arbitrary quantization schemes over general symplectic manifolds. Thus this algorithm can be applied to a wide variety of integrable systems appearing in different areas of mathematical physics (see 17-22]).

\section{References}

[1] V. I. Arnold, Mathematical Methods of Classical Mechanics (Nauka, Moscow, 1974; Springer, New York, 1978).

[2] A. T. Fomenko, Symplectic Geometry. Methods and Applications (Moscow State Univ., Moscow, 1988) (in Russian). 
[3] V. P. Maslov, Theory of Perturbations and Asymptotic Methods (Moscow State Univ., Moscow, 1965) (in Russian).

[4] M. Planck, Vorlesungen über die Theorie der Wärmestrahlung (Leipzig, 1906) (in German);

M. Planck, "Die physikalische Struktur des Phasenraumes," Annalen der Physik 50, 385-418 (1916) (in German).

[5] A. Einstein, "Zum Quantensatz von Sommerfeld und Epstein," Verhandl. Dtsch. Phys. Ges. 19, 82-92 (1917) (in German).

[6] P. Dirac, "The quantum algebra," Proc. Cambridge Phil. Soc. 23, 412-418 (1926).

[7] F. Berezin, "Quantization," Izv. Akad. Nauk SSSR Ser. Mat. 38 (5), 1116-1175 (1974) (in Russian).

[8] F. Bayen, M. Flato, C. Fronsdal, A. Lichnerowicz, and D. Sternheimer, "Deformation theory and quantization," Ann. of Phys. 111, 61-151 (1978).

[9] M. V. Karasev and V. P. Maslov, "Asymptotic and Geometric Quantization," Uspekhi Mat. Nauk 39 (6), 115-173 (1984) [Math. Surveys 39, 133-205 (1984)].

[10] H. Omori, Y. Maeda, and A. Yoshioka, "Weyl manifolds and deformation quantization," Adv. Math. 85, 224-255 (1991).

[11] M. Rieffel, "Deformation quantization for actions of $\mathbb{R}^{d}$," Mem. Amer. Math. Soc. 106, 1-93 (1993).

[12] V. V. Kozlov, Symmetries, Topology, and Resonances in Hamiltonian Mechanics (Izhevsk, 1995) (in Russian).

[13] M. V. Karasev and V. P. Maslov, Nonlinear Poisson Brackets. Geometry and Quantization (Nauka, Moscow, 1991) (in Russian).

[14] V. P. Maslov, "Semiclassical asymptotics for solutions of some problems in mathematical physics," Zh. Vychisl. Mat. i Mat. Fiz. 1, 113-128, 638-663 (1961) (in Russian).

[15] Y. Colin de Verdiere, "Spectre conjoint d'operateurs pseudodifferentiels qui commutent. I", Duke Math. J. 46, 169-182 (1979); II, Math. Z. 171, 51-73 (1980).

[16] M. V. Karasev, "Asymptotics of eigenvalues for operators with Poisson symmetry algebra of prime symbols," Funktsional. Anal. i Prilozhen. 18, 65-66 (1984) (in Russian).

[17] S. P. Novikov, "Hamiltonian formalism and multivalue analog of the Morse theory", Uspekhi Mat. Nauk 37, 3-49 (1982) (in Russian).

[18] L. A. Takhtadzhyan and L. D. Faddeev, Hamiltonian Approach in Soliton Theory (Nauka, Moscow, 1986) (in Russian). 
[19] M. A. Olshanetsky and A. M. Perelomov, Quantum Integrable Systems Related to Lie Algebras (Springer, 1983).

[20] Zhang-Ju Liu, "Quantum integrable systems constrained on the sphere," Lett. Math. Phys. 20, 151-157 (1990).

[21] P. P. Kulish, N. Manojlovich, and H. Samtleben, Infinite Dimensional Algebras and Quantum Integrable Systems (Springer, 2005).

[22] N. A. Slavnov, "Algebraic Bethe ansatz and quantum integrable systems", Uspekhi Mat. Nauk 62, 91-132 (2007) (in Russian). 